\title{
Computation of Mean Cerebral Blood Flow Velocity for the Assessment of Cerebral Autoregulation: Comparison of Different Strategies
}

\author{
Emanuele Vaini ${ }^{1}$, Vlasta Bari ${ }^{1}$, Davide Tonon ${ }^{2}$, Beatrice Cairo ${ }^{3}$, Beatrice De Maria ${ }^{4}$, Luca Faes ${ }^{5}$, \\ Gianluca Rossato ${ }^{2}$, Alberto Porta ${ }^{1,3}$ \\ ${ }^{1}$ Department of Cardiothoracic, Vascular Anesthesia and Intensive Care, IRCCS Policlinico San Donato, \\ San Donato Milanese, Milan, Italy \\ ${ }^{2}$ Department of Neurology, Sacro Cuore Hospital, Negrar, Verona, Italy \\ ${ }^{3}$ Department of Biomedical Sciences for Health, University of Milan, Milan, Italy \\ ${ }^{4}$ IRCCS Istituti Clinici Scientifici Maugeri, Milan, Italy \\ ${ }^{5}$ Department of Engineering, University of Palermo, Italy
}

\begin{abstract}
Cerebral autoregulation (CA) is a complex mechanism stabilizing cerebral blood flow $(C B F)$ against arterial pressure (AP) changes. $C B F$ is commonly surrogated with the $C B F$ velocity $(C B F V)$ recorded via transcranial Doppler device from the middle cerebral artery. Most of the studies evaluating CA compute mean CBFV (MCBFV) on a beat-to-beat basis along with mean AP (MAP), but there is not a standard approach to derive MCBFV. In this study, we compare three different strategies to calculate MCBFV: i) between two consecutive diastolic points detected on the $C B F V$ signal $\left(M C B F V_{C B F V}\right)$; ii) between two consecutive diastolic points detected on the $A P$ signal $\left(M C B F V_{A P}\right)$; iii) between two consecutive $R$ wave peaks detected on the ECG $\left(M C B F V_{E C G}\right)$. We analyzed ECG, noninvasive $A P$ and $C B F V$ signals recorded from 23 subjects (age: $28 \pm 9$ yrs, 13 female) at rest in supine position (REST) and during head-up tilt at $60^{\circ}$ (TILT). While means were similar regardless of the considered strategy, variances significantly varied with $M C B F V_{C B F V}$ and $M C B F V_{E C G}$ strategy producing the largest and the smallest variance respectively. This result stresses the need to standardize the approach for MCBFV computation to reduce the variability of the results solely due to the method adopted for its computation and favor clinical applications of CA assessment.
\end{abstract}

\section{Introduction}

The evaluation of cerebral autoregulation (CA) necessitates the recording of arterial pressure (AP) and cerebral blood flow (CBF) [1]. The AP is monitored in dynamic conditions with a high accuracy and adequate temporal resolution using noninvasive photoplethysmographic devices [2,3]. The CBF is usually approximated via $\mathrm{CBF}$ velocity (CBFV) recorded from the middle cerebral artery via transcranial Doppler device under the assumption that the diameter of insonated vessels remains constant during the measurement [4]. The assessment of CA often relies on signal processing techniques applied to the spontaneous variations of mean AP (MAP) and mean CBFV (MCBFV) [5] and this evaluation has been found useful for the assessment of cerebrovascular control in physiological and pathological conditions [6-8].

The evaluation of a mean value of a signal in an assigned period of time requires the identification of two fiducial points providing the onset and the offset for computation of the definite integral. Different strategies were followed to compute MCBFV on a beat-to-beat basis and these strategies depended on the selection of the two fiducial points. While in some studies the diastolic points were detected directly on CBFV signal [9-12], in other research papers the occurrence of the diastolic events was taken from the AP signal [13]. Some studies exploited the R-wave peak of the electrocardiogram (ECG) to define the temporal limits for the computation of the current MCBFV over CBFV [14]. However, AP, CBFV and ECG are not immune by artifacts that differently affect the three signals leading to diverse signal-to-noise ratios. For example, the CBFV recording obtained via transcranial Doppler device depends on several geometrical factors, such as the probe insonation angle, dramatically influenced by patient's and technician's movements [15], while a typical physiological artifact of the photoplethysmographic AP signal, strongly influencing diastolic points, is the peripheral vasomotion [16]. The ECG signal is strongly affected by thoracic muscle activity and cardiac axis movements linked to respiration [17]. As a result of these influences, the accuracy of the MCBFV measurement might depend on the signal-to-noise ratio of the signal providing the two fiducial points and this dependence might affect the reliability of the CA assessment. The lack of standardization in the MCBFV computation is not only an issue for comparison different studies, but it could also represent a problem for the CA measurement accuracy.

The aim of this study is to test three different strategies 
to extract MCBFV measures on a beat-to-beat basis in a population of subjects at rest and during a postural challenge. Time domain indexes of MCBFV were computed according to whether the fiducial points for the computation of MCBFV were extracted from CBFV, AP and ECG respectively.

\section{Experimental protocol and analysis}

\subsection{Experimental protocol}

The study took place at the Neurology Department of Sacro Cuore Hospital, Negrar, Italy, and was conducted in keeping with Helsinki Declaration for studies regarding human subjects after the approval of the local ethics committee. A written informed consent was signed by each subject before being admitted to the study.

Experimental protocol was fully described elsewhere [10]. Briefly, we recorded ECG, noninvasive AP through a photoplethysmographic device (Finapres Medical Systems, Ohmenda, The Netherlands) and CBFV from the middle cerebral artery using a transcranial Doppler device (Multi-Dop T2, Dwl, San Juan Capistrano, CA) in from 23 subjects (age: $28 \pm 9$ yrs, 13 female). Experimental protocol consisted in 10 minutes at rest in supine position (REST) followed by 10 minutes of headup tilt with tilt table inclination at $60^{\circ}$ (TILT). Signals were sampled at $1000 \mathrm{~Hz}$. The CBFV signal was lowpass filtered with a sixth-order Butterworth filter with cut-off frequency of $10 \mathrm{~Hz}$, and attention was paid to avoid any phase distortion [10].

\subsection{Strategies for MCBFV computation}

MCBFV was computed as the ratio of the definite integral of the CBFV signal calculated between two fiducial points to the time interval between them. MCBFV was expressed in $\mathrm{cm} \cdot \mathrm{s}^{-1}$. Three MCBFV measures were performed and the measures varied with the strategy adopted for the selection of the two fiducial points for the computation of the definite integral. While in the first and second measure, termed $\mathrm{MCBFV}_{\mathrm{CBFV}}$ and $\mathrm{MCBFV}_{\mathrm{AP}}$, the two fiducial points were taken in correspondence of two consecutive diastolic points detected on the CBFV and AP signals respectively, in the third measure, labelled $\mathrm{MCBFV}_{\mathrm{ECG}}$, the two fiducial points were identified by the occurrence of two consecutive R-wave peaks on the ECG.

\subsection{Time domain and statistical analysis}

MAP was computed as the ratio of the definite integral of the AP signal calculated between two diastolic points to the interdiastolic time interval. MAP was expressed in $\mathrm{mmHg}$. Heart period (HP) was computed as the time
Table 1. Time domain indexes from HP and MAP series.

\begin{tabular}{ccc}
\hline Parameter & REST & TILT \\
\hline$\mu_{\mathrm{HP}}[\mathrm{ms}]$ & $869 \pm 173$ & $699 \pm 114^{*}$ \\
$\sigma^{2} \mathrm{HP}\left[\mathrm{ms}^{2}\right]$ & $3036 \pm 3229$ & $1991 \pm 1454$ \\
$\mu_{\mathrm{MAP}}[\mathrm{mmHg}]$ & $93.7 \pm 16.7$ & $97.3 \pm 15.4$ \\
$\sigma_{\mathrm{MAP}}^{2}\left[\mathrm{mmHg}^{2}\right]$ & $15.9 \pm 17.3$ & $18.7 \pm 10.2$ \\
\hline
\end{tabular}

$\mu_{\mathrm{HP}}=$ HP mean; $\sigma_{\mathrm{HP}}^{2}=$ HP variance; $\mu_{\mathrm{MAP}}=$ MAP mean; $\sigma^{2}$ MAP $=$ MAP variance; REST $=$ at rest in supine position; TILT $=$ head-up tilt. The symbol $*$ indicates $p<0.05$ versus REST.

distance between two consecutive R-wave peaks on the ECG. HP was expressed in ms. HP, MAP, and the three MCBFV values were taken on a beat-to-beat basis. The resulting beat-to-beat series were manually inspected and corrected in case of misdetection or isolated arrhythmic events through linear interpolation. The rate of corrections was less than $5 \%$ of the total length of beatto-beat series. In agreement with the guidelines for shortterm analysis of cerebrovascular control, synchronous sequences lasting 250 consecutive beats were randomly selected from entire series recorded during the session. TILT sequences were selected after 5 minutes from the onset of the TILT session. For each sequence, time domain markers, such as mean and variance, namely $\mu_{\mathrm{HP}}$, $\mu_{\mathrm{MAP}}, \mu_{\mathrm{MCBFV}, \mathrm{CBFV}}, \mu_{\mathrm{MCBFV}, \mathrm{AP},} \mu_{\mathrm{MCBFV}, \mathrm{ECG}}$ and $\sigma_{\mathrm{HP}}^{2}, \sigma^{2} \mathrm{DAP}$, $\sigma^{2}{ }_{M C B F V, C B F V}, \sigma^{2}{ }_{M C B F V}, \mathrm{AP}, \sigma^{2}{ }_{M C B F V, E C G}$ were calculated from the series of HP, MAP, MCBFV $\mathrm{CBFV}_{1}, \mathrm{MCBFV}_{\mathrm{AP}}$ and $\mathrm{MCBFV}_{\mathrm{ECG}}$ respectively. Variances were calculated after linear detrending.

\subsection{Statistical analysis}

Two-way analysis of variance (Holm-Sidak correction for multiple comparisons) was performed over time domain indexes to evaluate the significance of the between-condition differences within the same type of MCBFV measure and between-marker within the same experimental condition. Statistical analysis was carried out using a commercial statistical sofware (Sigmaplot, v.14.0, Systat Software, Inc., Chicago, IL). A $p<0.05$ was always considered statistically significant.

\section{Results}

Table 1 shows the time domain indexes computed over HP and MAP series extracted at REST and during TILT. Orthostatic challenge reduced $\mu_{\mathrm{HP}}$, while $\mu_{\mathrm{MAP}}, \sigma_{\mathrm{HP}}^{2}$ and $\sigma^{2}$ MAP were unmodified. Figure 1 shows the time domain indexes computed over MCBFV, namely $\mu_{\mathrm{MCBFV}}$ (Fig.1a) and $\sigma^{2}{ }_{M C B F V}$ (Fig.1b), as a function of the experimental condition (i.e. REST and TILT). MCBFV measures were computed over $\mathrm{MCBFV}_{\mathrm{CBFV}}$ (black bars), $\mathrm{MCBFV}_{\mathrm{AP}}$ (light gray bars) and $\mathrm{MCBFV}_{\mathrm{ECG}}$ (dark gray bars) series according to the three different strategies of measurement. 

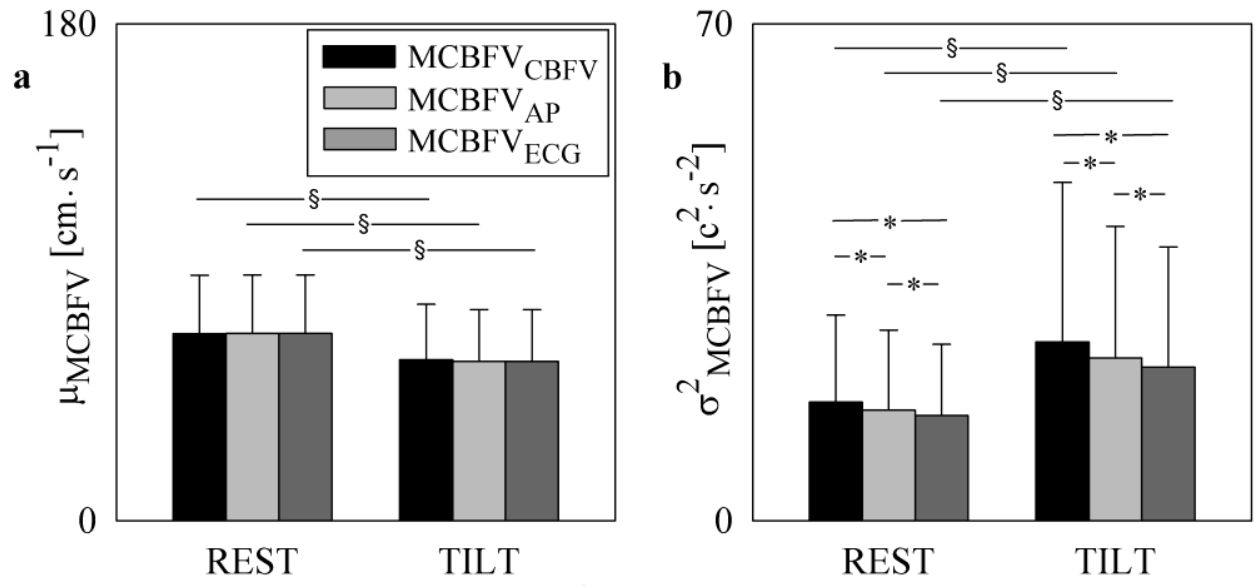

Figure 1. The grouped bar graphs show $\mu_{\mathrm{MCBFV}}$ (a) and $\sigma^{2} \mathrm{MCBFV}$ (b) computed at REST and during TILT. MCBFV makers were computed according to the three different strategies, namely $\mathrm{MCBFV}_{\mathrm{CBFV}}$ (black bars), $\mathrm{MCBFV}_{\mathrm{AP}}$ (light gray bars) and $\mathrm{MCBFV}_{\mathrm{ECG}}$ (dark gray bars). The symbol * indicates $p<0.05$ versus $\mathrm{MCBFV}_{\mathrm{CBFV}}$ measures within the same condition (REST or TILT). The symbol $\S$ indicates $p<0.05$ versus REST within the same strategy for the MCBFV assessment.

Regardless of the strategy, $\mu_{\mathrm{MCBFv}}$ decreased and $\sigma^{2} \mathrm{MCBFV}$ increased during TILT. Assigned the experimental condition $\mu_{\mathrm{MCBFV}}$ did not vary with the strategy of measurement. Conversely, $\sigma^{2} \mathrm{MCBFV}$ depended on the method exploited to compute MCBFV both at REST and during TILT reaching the maximum value when computed over the $\mathrm{MCBFV}_{\mathrm{CBFV}}$ series and the minimum value when calculated over the $\mathrm{MCBFV}_{\mathrm{ECG}}$ series.

\section{Discussion}

The assessment of CA from spontaneous slow fluctuations of AP and CBFV is based on the evaluation of the MCBFV variability and its dynamical interactions with MAP variations [5,7,10-13]. Therefore, the CA quantification could vary whether MCBFV variability depended on the strategy adopted for its computation. Our study suggests that, while the mean of MCBFV is independent of the technique exploited for the MCBFV assessment, the variance of MCBFV differs significantly.

The computation of MCBFV during each cardiac cycle is crucial for the CA assessment [12]. The calculation of the MCBFV requires the computation of a definite integral over the CBFV signal between two fiducial points defining the starting and ending instants for the computation of the area under the CBFV curve. In literature there is no agreement on the definition of these two fiducial points. Some studies proposed to exploit the time at the CBFV minimum (i.e. diastolic time), thus using exclusively the CBFV signal for the computation of MCBFV [9-12]. Conversely, other research papers detected the diastolic time over the AP signal [13] in correspondence of the AP diastole, thus necessitating the acquisition of AP in addition to CBFV. Finally, some reports exploited the easiness of delineating the R-wave peak on the ECG to fix the reference times defining the onset and offset of the current cardiac cycle [14]. The strategy adopted for the computation of the MCBFV did not affect its mean value given that the mean of $\mathrm{MCBFV}_{\mathrm{CBFV}}, \mathrm{MCBFV}_{\mathrm{AP}}$ and $\mathrm{MCBFV}_{\mathrm{ECG}}$ were similar. Conversely, the variance of the MCBFV varied systematically with the method utilized to compute MCBFV. More specifically, the method exploiting the diastolic time detected on the CBFV signal produced MCBFV estimates with the largest variance, while the approach using the R-wave peaks delineated on the ECG led to the smallest variance. The MCBFV measure obtained by the method using the diastolic points found over the AP signal featured intermediate values of variance. Remarkably, the systematic MCBFV variance differences held irrespective of the experimental condition.

The fiducial points utilized for the computation of the definite integral on the CBFV signal jitter in relation to measurement noise and physiological factors. Measurement noise is mainly related to contaminating factors, such as instrumentation noise, usually quantified by the signal-to-noise ratio. For example, all the considered signals are strongly influenced by motion artifacts generated by movements of the ultrasonographic probe, finger cuff, and electrodes in the case of CBFV, noninvasive AP, and ECG respectively. Moreover, also physiological influences, not directly related with the variables being monitored, can generate measurement artifacts such as motion of the wall of the middle cerebral artery in relation to the pulsatile nature of the CBF [15], modifications of peripheral resistances governed by the sympathetic arm of baroreflex directly affecting the diastolic runoff of the AP [16], cardiac axis movements synchronous with respiration altering ECG waveforms [17]. Measurement noise and physiological factors could produce constant biases in the position of the fiducial points utilized for the computation of MCBFV as well as affect the amplitude of the jitters about their mean 
position. These effects might be dependent on the strategy adopted for the computation of the MCBFV. Since the MCBFV mean is stable with the approach, we conclude that measurement noise and physiological factors affecting differently CBFV, AP and ECG do not produce constant biases given that it is unlikely that they cause the same bias over all the signals. Conversely, since the MCBFV variance varies with the strategy, the amplitude of the jitters depends on the signal where the fiducial points are delineated. Since the signal with the worst signal-to-noise ratio is the CBFV one and the ECG signal is the one with the best signal-to-noise ratio, we interpret the differences of the MCBFV variance with the method as a consequence of the impact of noise increasing the variability of the fiducial points about their average position. Therefore, for the computation of MCBFV we recommend the delineation of the fiducial points on the ECG to minimize the variance of the MCBFV estimates.

\section{Conclusions}

Different techniques for the computation of MCBFV lead to similar mean values but different variances. This finding should not be ignored when the practical aim is the characterization of CA based on MCBFV and MAP spontaneous fluctuations. We recommend the strategy computing the definite integral over the CBFV signal between two consecutive R-wave peaks detected on the ECG. This strategy allows the greatest reduction of the MCBFV variability likely in relation to the greatest signal-to-noise ratio of ECG signal compared to those of the CBFV and AP ones. By limiting the MCBFV variability this strategy could help the separation among different experimental conditions within the same population and the distinction of diverse populations within the same experimental condition. We recommend also the standardization of the MCBFV measurement procedures to favor comparison among different studies and we stress the need of reporting the strategy for the MCBFV computation in any report about CA.

\section{References}

[1] O. B. Paulson, S. Strandgaard and L. Edvinsson, "Cerebral autoregulation," Cerebrovasc. Brain Metab. Rev., vol. 2, pp. 161-192, 1990.

[2] G. Parati et al., "Non-invasive beat-to-beat blood pressure monitoring: new developments," Blood Press. Monit., vol. 8, pp. 31-36, 2003.

[3] B. P. Imholz, W. Wieling, G. A. van Montfrans and K. H. Wesseling, "Fifteen years experience with finger arterial pressure monitoring: assessment of the technology," Cardiovasc. Res., vol. 38, pp. 605-616, 1998.

[4] R. Aaslid, T. M. Markwalder and H. Nornes, "Noninvasive transcranial Doppler ultrasound recording of flow velocity in basal cerebral arteries," J. Neurosurg., vol. 57, pp. 769774, 1982.
[5] R. B. Panerai, S. L. Dawson, P. J. Eames and J. F. Potter, "Cerebral blood flow velocity response to induced and spontaneous sudden changes in arterial blood pressure," Am. J. Physiol., vol. 280, pp. H2162-H1274, 2001

[6] A. T. Yam et al., "Cerebral autoregulation and ageing," $J$. Clin. Neurosci., vol. 12, pp. 643-646, 2005.

[7] V. Bari et al., "Cerebrovascular and cardiovascular variability interactions investigated through conditional joint transfer entropy in subjects prone to postural syncope," Physiol. Meas., vol. 38, pp. 976-991, 2017.

[8] R. B. Panerai, "Cerebral autoregulation: from models to clinical applications," Cardiovasc. Eng., vol. 8, pp. 42-59, 2008.

[9] E. Vaini et al., "Causality analysis reveals the link between cerebrovascular control and acute kidney dysfunction after coronary artery bypass grafting", Physiol. Meas., 40, 064006, 2019

[10] V. Bari et al., "Nonlinear effects of respiration on the crosstalk between cardiovascular and cerebrovascular control systems," Philos. Trans. R. Soc. A., vol. 374, 20150179, 2016.

[11] L. Faes et al., "Investigating the mechanisms of cardiovascular and cerebrovascular regulation in orthostatic syncope through an information decomposition strategy," Auton. Neurosci., vol. 178, pp. 76-82, 2013.

[12] R. Zhang et al., "Transfer function analysis of dynamic cerebral autoregulation in humans," Am. J. Physiol., vol. 274, pp. H233-H241, 1998.

[13] J. A. Claassen, A. S. Meel-van den Abeelen, D. M. Simpson, R. B. Panerai and international Cerebral Autoregulation Research Network (CARNet), "Transfer function analysis of dynamic cerebral autoregulation: A white paper from the International Cerebral Autoregulation Research Network," J. Cereb. Blood Flow Metab., vol. 36, pp. 665-680, 2016.

[14] E. Katsogridakis et al., "Signal-to-noise ratio of bilateral nonimaging transcranial Doppler recordings of the middle cerebral artery is not affected by age and sex," Ultrasound Med. Biol., vol. 37, pp. 530-538, 2011.

[15] Q. Shen, J. Stuart, B. Venkatesh, J. Wallace and J. Lipman, "Inter observer variability of the transcranial Doppler ultrasound technique: impact of lack of practice on the accuracy of measurement," J. Clin. Monit. Comput., vol. 15, pp. 179-184, 1999.

[16] A. Porta et al., Peripheral resistance baroreflex during incremental bicycle ergometer exercise: characterization and correlation with cardiac baroreflex, Front. Physiol., 9, 688, 2018.

[17] A. Porta et al., "Performance assessment of standard algorithms for dynamic R-T interval measurement: comparison between R-Tapex and R-Tend approach," Med. Biol. Eng. Comput., vol. 36, pp. 35-42, 1998.

Address for correspondence:

Dr Emanuele Vaini

IRCCS Policlinico San Donato

Via F.Fellini 4, 20097 San Donato Milanese, Milan, Italy

E-mail: emanuele.vaini@grupposandonato.it 